\title{
Development of a pattern recognition algorithm for particle identification on the SciCRT in the Sierra Negra Volcano Summit
}

\author{
Rocío García $^{* a}$, J.F.Valdés-Galicia ${ }^{a}$, Marcos Anzorena ${ }^{a}$, Ernesto Ortiz ${ }^{b}$, \\ L.X.González ${ }^{c}$,Octavio Musalem ${ }^{a}$, Alejandro Hurtado ${ }^{a}$, Marco Barrantes $^{a}$, Roberto \\ Taylor $^{a}$, Yutaka Matsubara $^{d}$, Yoshinori Sasai $^{d}$, Yoshitaka Itow $^{d}$, Takashi Sako $^{d}$, Tetsuya \\ Kawabata $^{d}$, Akira Tsuchiya $^{d}$, Kazuoki Munakata $^{e}$,Chihiro Kato $^{e}$, Yoshiaki \\ Nakamura $^{e}$,Takahiro Oshima $^{e}$, Toshiki Koike $^{e}$, Shoichi Shibata $^{f}$, Akitoshi Oshima $^{f}$, \\ Hisanori Takamaru ${ }^{f}$, Hiroshi Kojima ${ }^{g}$, Harufumi Tsuchiya $^{h}$, Kyoko Watanabe $^{i}$, \\ Masayoshi Kozai ${ }^{j}$, Tatsumi Koi ${ }^{k}$. \\ E-mail: rociodgeofisica.unam.mx
}

\begin{abstract}
At the top of the Sierra Negra volcano in eastern México $\left(19.0^{\circ} \mathrm{N}, 97.3^{\circ} \mathrm{W}\right)$ the SciBar Cosmic Ray Telescope (SciCRT) is installed, one of its main purposes is to detect solar neutrons to investigate the ion acceleration process during intense solar flares. Furthermore, thanks to the design and construction of the SciCRT in the form of small and long scintillation bars, large active volume, high energy resolution, and a fast electronics for data processing, particle identification is possible through the analysis of tracks. Considering these properties, species identification of secondary cosmic ray inside the Earth's atmosphere, at a depth about $600 \mathrm{~g} \mathrm{~cm}^{-2}$ is possible. In this work, we present an ad-hoc algorithm constructed to distinguish between particle species that cross the active volume of the detector. The aim is to use pattern recognition methods and event reconstruction to achieve this goal.
\end{abstract}

35th International Cosmic Ray Conference

10-20 July, 2017

Bexco, Busan, Korea

\footnotetext{
* Speaker.

${ }^{\dagger a}$ Instituto de Geofísica,Universidad Nacional Autónoma de México,Coyoacán,CDMX, 04510, México.

${ }^{\dagger}$ Instituto de Ciencias Físicas,Universidad Nacional Autónoma de México, Cuernavaca, Morelos,62210, México.

${ }^{\dagger c}$ SCiESMEX, Instituto de Geofísica, Unidad Michoacán, Universidad Nacional Autónoma de México, Michoacán Morelia, 58190, México.

${ }^{\dagger d}$ Institute for Space-Earth Environmental Research, Nagoya University, Furo-cho, Chikusa-ku, Nagoya 4648601,JAPAN
} 


\section{Introduction}

Driven by the desire to study and understand the cosmic ray radiation, the scientists have developed radiation detectors with many different characteristics. The design of these instruments depend on a large extent on the information that we want to obtain from the radiation.

So far, the Institute of Geophysics of UNAM has three types of detectors to study different aspects of cosmic radiation, two of them in an international collaboration.

In the cosmic ray observatory at Mexico City there is a Neutron Monitor (NM64), working since 1990. The key operating principle of NM64 is the nuclear reaction inside proportional counters, making it capable to measure the variation of cosmic ray intensity.

On the other hand, at the top of the Sierra Negra volcano in Mexico, two detectors were installed in order to study solar neutron phenomena. One of them, the Solar Neutron Telescope (SNT), consists of an array of proportional counters working as veto counters, and plastic scintillators capable of registering the energy from incident incident radiation. In addition, this telescope also provides a means to measure the intensity of cosmic rays components and arrival direction[1]. However, as much as the energy and spatial resolution of the SNT is suitable for solar neutron detection, there are still many open questions regarding the particle acceleration mechanisms at the Sun. This was our main motivation to install an improved solar neutron telescope.

The SciBar Cosmic Ray Telescope (SciCRT)[2] is a new detector with better energy resolution making use of scintillator bars of rectangular shape $(1.3 \mathrm{~cm} \times 2.5 \mathrm{~cm} \times 300 \mathrm{~cm})$ organized in layers stack up perpendicular, we have a good spatial resolution. Every layer is composed of 112 scintillator bars. A schematic description is shown in Figure 1. Furthermore, thanks to SciCRT's large volume (from 14.848 bars) the estimation of the Energy loss per unit length $(-d E / d x)$ allows the identification of particle species.

An array of 16 layers of scintillator bars ( 8 on the $\mathbf{X}$ direction and 8 on the $\mathbf{Y}$ direction) receives the name of super block (SB). The SciCRT has in total eight SBs. One SB allows to obtain one data image per side, taken from the interaction of particles with the detector.

The SciCRT is constructed from scintillation bars. When the incident radiation interacts with the atoms of the bars, it transfers kinetic energy. As a result, the excited atoms emit photons when they return to their ground state. After, the photons produced are absorbed and remitted by wavelength shifting (WLS) fibers. The main purpose is to guide the photons from the scintillator bars to the photodetector installed on one end of the detector.

The multi-anode photomultiplier tubes (MAPMTs) convert the photons into electrons through the process of photoelectric emission in the photocathode material, these are used to estimate the energy deposit on the scintillators. A fast costume made data acquisition system[3] is capable of reading the output from each MAPMT and to discriminate between muons and neutral particles.

\footnotetext{
${ }^{e}$ Department of Physics, Shinshu University, Asahi, Matsumoto 390-8621, JAPAN.

${ }^{f}$ College of Engineering, Chubu University, Kasugai 487-8501, JAPAN.

${ }^{g}$ Faculty of Engineering, Aichi Institute of Technology, Toyota 470-0392, JAPAN.

${ }^{h}$ Japan Atomic Energy Agency, 2-4 Shirakata Shirane, Tokai-mura, Naka-gun, Ibaraki 319-1195, JAPAN.

${ }^{i}$ National Defense Academy of Japan, 1-10-20 Hashirimizu, Yokosuka, Kanagawa 239-8686, JAPAN.

${ }^{j}$ Institute of Space and Astronautical Science, Japan Aerospace Exploration Agency, Sagamihara, Kanagawa 2525210, Japan.

${ }^{k}$ SLAC National Accelerator Laboratory, Menlo Park, CA 94025-7015, USA
} 

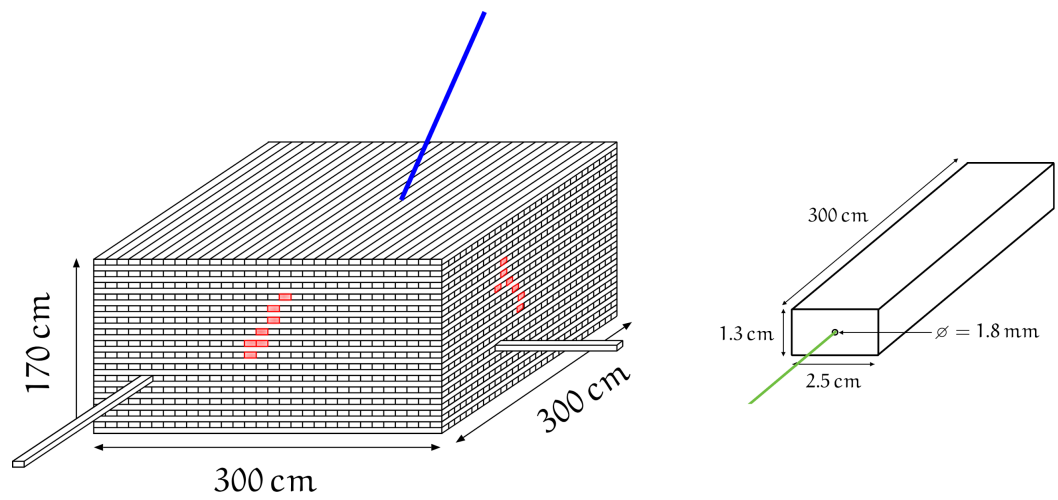

Figure 1: Schematic diagram of SciBar Cosmic Ray Telescope SciCRT.

In this work, we present an alternative algorithm constructed ad-hoc to distinguish between particle species that cross the active volume of the detector. With this study, we may estimate the percentage of different particle species detected by the neutral channel in the SciCRT. At the same time, we develop a method for estimating the direction and total energy deposition.

\section{Methodology}

Pattern recognition has a wide range of applications, including speech recognition, electrocardiogram analysis, scene analysis and of course many high-energy physics experiments. In general, the pattern recognition can be defined as[4]:

Given $n$ measurements of an object (the raw data of a high-energy physics event), one wants to associate this specific object with one out of several classes of objects. If $x$ is the vector consisting of all measurement values, one is therefore looking for a decision function $d$ such that

$$
c=d(x)
$$

Where $c$ is the class to which $x$ belongs. In the general case, classes may share objects, but in high-energy physics we are mainly interested in exclusive classes, which do not share objects.

\subsection{Track Finding and fitting}

The first step in the methodology is the track finding. At first, we evaluate two possible methods: template matching and Hough Transform. Both are classified in the literature like global methods[5]. These have the common property of treating all hit information in an equal and unbiased way. The simultaneous considerations of all hits can be very inefficient in terms of speed[6], however this is not significant since we do not want to achieve real-time computing.

The purpose is to find a subset called track candidates, that is, each subset contain measurements believed to be originated from the same particle.

\subsubsection{Template matching}

This method requires a dictionary of all possible tracks. In order to test this method we need a reference track model. These will be generated by means of a Monte Carlo simulation. Due to the 
physics motivation of our experiment, we focus first in the interactions by neutrons, protons and $\gamma$-rays on the detector.

However, template matching does not scale well when the required spatial resolution is fine. As a result, the number of templates exceeds the limits of feasibility when storing is of concern or when the number of steps increases significantly.

A possible solution is the tree-search algorithm[4], this uses templates with different resolution, ordered in hierarchy. In others words, we can first match a small set of patterns with coarse resolution and in the next step, a second set of templates with a better resolution is possible.

\subsubsection{Hough Transform}

The track finding process often uses the knowledge of how a particle moves inside the detector. With the Hough Transform[7] we can find all types of tracks of different shapes, as well as; circle, ellipse, line, always that it can be describe with a mathematical model.

In the SciCRT, we will only find tracks that are close to straight lines. For this reason we will apply the Hough transform to detect straight lines. A line can be described by:

$$
x \cos \phi+y \sin \phi=\rho
$$

The Hough space for lines has therefore two dimensions; $\theta$ and $\phi$, and a line is represented by a single point, corresponding to a unique set of parameters $\left(\phi_{0}, \rho_{0}\right)$. The line-to-point mapping is illustrated in the right figure 2. This is an example of Hough transform on a muon track measure with sixteen layers of scintillator bars.

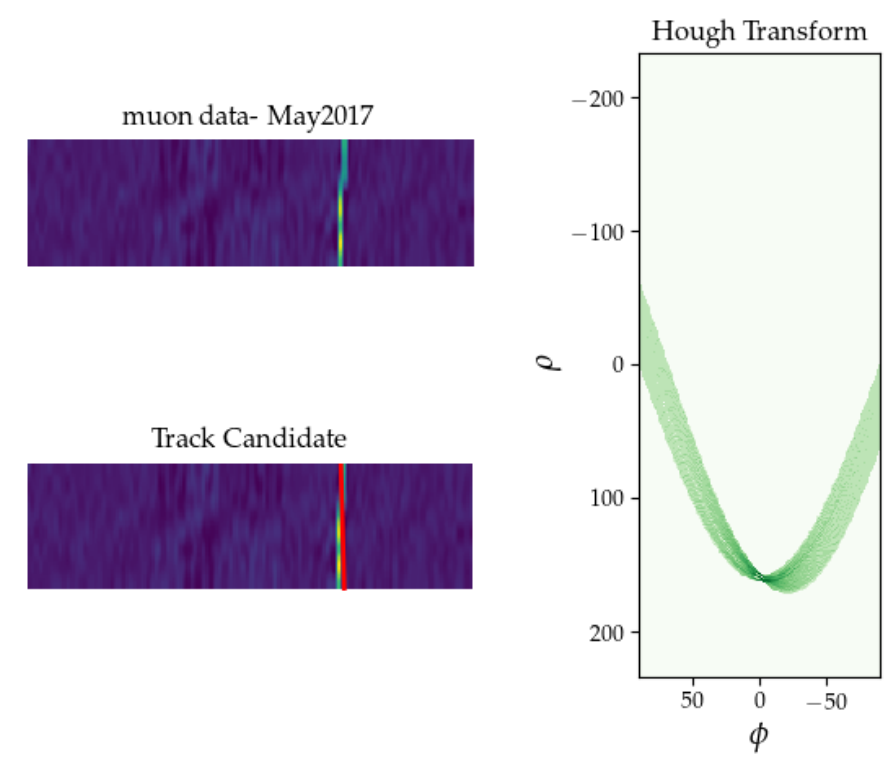

Figure 2: The Hough transform. The top-left panel shows a muon track crossing 16 layers of scintillator bars. The bottom panel shows the result of detection (red line) using the Hough Transform. The right panel is the Hough transform 


\subsubsection{Particle fitting}

In the particle fitting we analyze the data from all SBs, 2 images correspond to $\mathbf{X}$ and $\mathbf{Y}$ sides. The tracks from each side of the detector are fitted to a track model. The fit will allow to interpolate the tracks in the places where the detector is unable to record the passage of particles.

Strict fit criteria is prohibited by different physics phenomena, such as the occurrence of the detection of 2 or more tracks on the same event (track pile-up). For the task of track fitting, we use a Maximum Likelihood Method.

\subsection{Event Reconstruction}

Finally in the last step we want to reconstruct the particle's path inside of the SciCRT, joining together the $\mathbf{X}$ and $\mathbf{Y}$ fitting. Then, analyzing the way the particles interact we can use some methods for Particle Identification (PID).

At first, PID is possible by difference in interaction, for example, determine if they produce an electromagnetic or hadronic shower. Another method is the measurement of energy loss per unit path length $-d E / d x$ [8]. Moreover, if the particle deposited all its energy in the active volume of the detector, we can estimate the incident energy.

\section{Current Progress}

\subsection{Calibration by cosmic-ray muons}

In order to calculate the energy loss, we need an energy calibration to determine the absolute gain of each channel with high precision. The energy scale of each scintillator bar is calibrated via cosmic-ray muons.

Figure 3 shows the ADC distribution of muon from one channel. From the figure we can separate the distribution in two perceptible areas. The first area from the lower energy region corresponds to noise and crosstalk effect. The area in the higher energy region is the muon signal. The goal is to remove the noise signal and then calculate the relation between ADC value and deposition energy

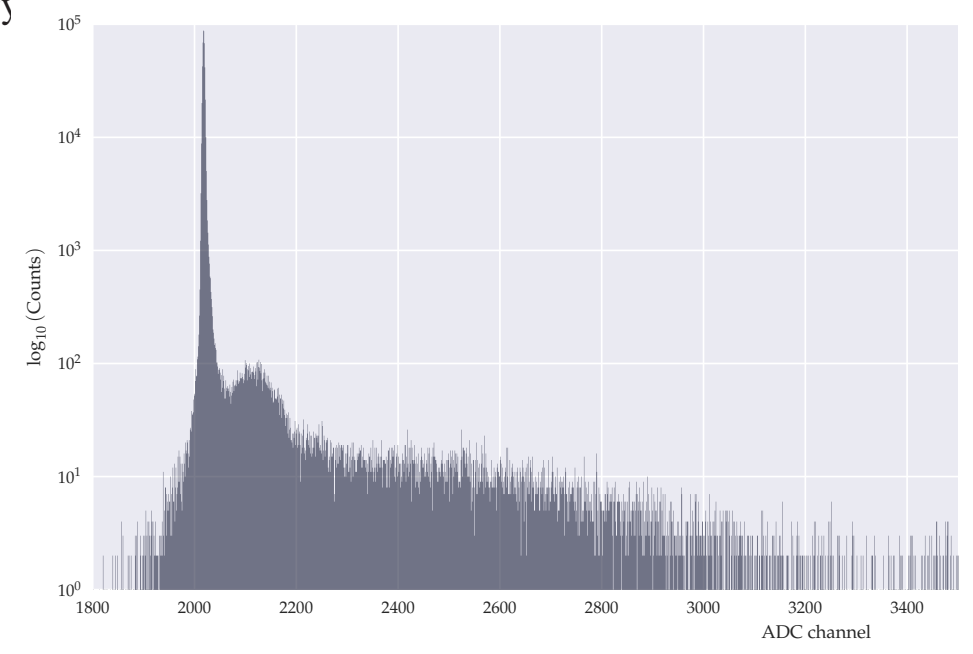

Figure 3: Muon $\mathrm{ADC}$ distribution at $\mathrm{SB}_{3}$. Data from March 2017 
We developed an algorithm to determine the ADC thresholds $\left(A D C_{t h}\right)$, the choice of an ADC value allows to separate cosmic rays from noise. The algorithm works by fitting a gaussian distribution to each peak. For fitting muon signal peak, we need to select data interval, with the use of some definied criteria. Also, the fit method estimate parameters like standard deviation. After, the algorithm makes a correlation analysis. We use the coefficient of determination (square of the correlation coefficient) to determines the extent of proportionality between two variables[9]. A correlation coefficient of 1 signifies perfect correlation.

After, the $A D C_{t h}$ will be the point of intersection of the two Gaussian distributions.

\subsection{Simulation}

As already mentioned above, a paramount ingredient of our methodology is the simulation of the detector. At the current time, we are working on the description of the scintillator bars using Geant4[10].

Extruded scintillator. The scintillator bar has a rectangular cross section of $2.5 \mathrm{~cm}$ wide and $1.3 \mathrm{~cm}$ thick and $300 \mathrm{~cm}$ length. In the center, it has a $1.8 \mathrm{~mm}$ diameter hole. The bar is covered with a co-extruded reflector material, it has $0.25 \mathrm{~mm}$ thick and contains $15 \%$ of $\mathrm{TiO}_{2}$ by weight in polystyrene. In the center, a wavelength shifting fiber of $1.5 \mathrm{~mm}$ diameter is installed. In the simulation we put a MAPMT at $20 \mathrm{~cm}$ from the bar.

\section{Result and Discussion}

In relation to calibration step, we process the data from 1200 channels. The gain of the MAPMT is defined by 4.1. The result of this calculation is shown on the figure 4. The figure takes into account only the channels that have $R^{2} \geqslant 0.8$. However, with this criteria about only the $50 \%$ of the channels process correctly. The problem may arise from the selection of interval above stated. We need further tests to find the proper method for this estimate.

$$
\text { Gain }=\frac{A D C_{t h}-m_{p}}{\text { mip }}
$$

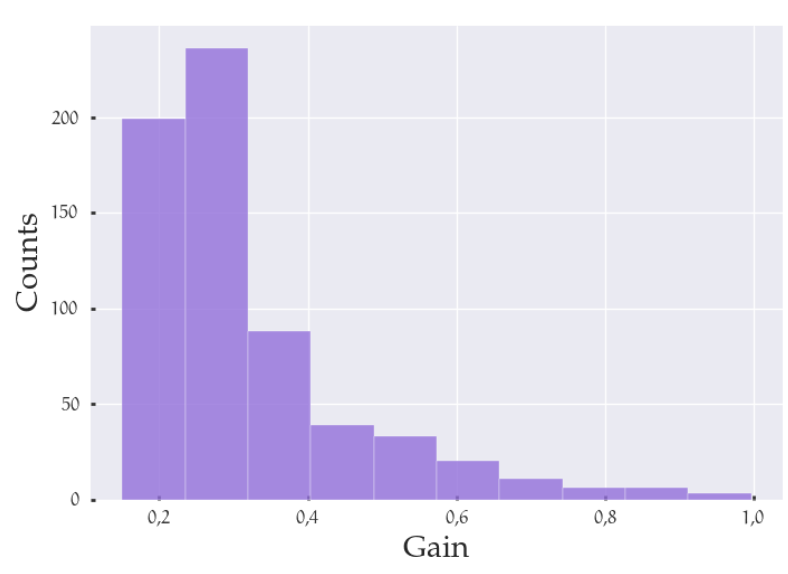

Figure 4: MAPMT gain distribution normalized by MIP value 
On the other hand, using the description of the scintillator bars, we inject 1500 muons crossing at the middle of the bar with different energies. Figure 5 shows an example of the energy loss distribution for muons with $650 \mathrm{MeV}$, the central tendency is $2.179 \times 10^{4}$ photons produced.

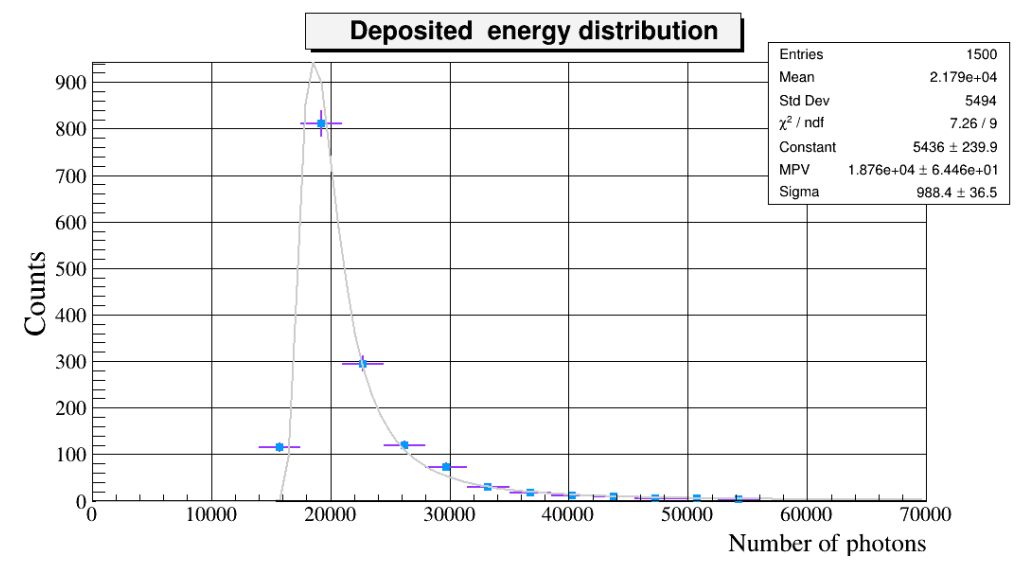

Figure 5: Deposited energy by $650 \mathrm{MeV}$ muons on one scintillator bar.

Figure 6 shows the behavior of energy losses for muons interacting with atoms of scintillator bars. As expected, around $400 \mathrm{MeV}$ the energy losses becomes almost constant.

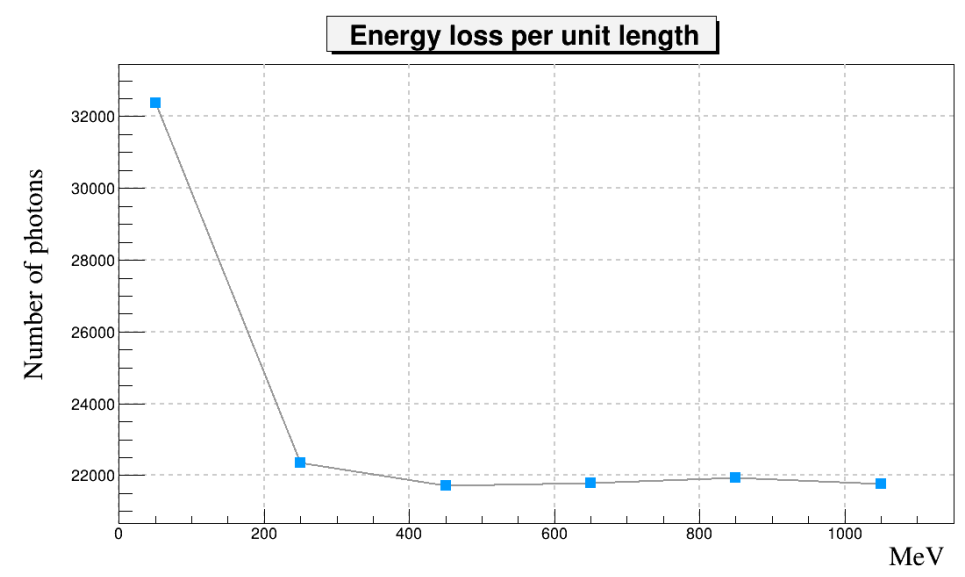

Figure 6: Energy loss per unit length for muons crossing $1.2 \mathrm{~cm}$ of scintillator material

The future tasks consists in programming the full description of the SciCRT, and get the track models in order to continue the test for pattern recognition methods, with the main goal of obtaining an adequate method designed for SciCRT. Also we need finish the characterization of all channels from MAPMT and make the analysis of energy deposition.

\section{Summary}

The SciBar Cosmic Ray Telescope (SciCRT) is a new detector of type scintillation detector, it has a large active volume and good spatial resolution. These features make the SciCRT ideal for applying pattern recognition methods. Some tools such as Hough transform, Template Matching, 
among others, can be used to analyze the cosmic ray radiation arriving to the detector and make possible the identification different species of particles.

\section{Acknowledgments}

This work is partially supported by UNAM-PAPIIT-IN104115 and CONACyT-180727T. We also are acknowledged Grants from Scientific Research(B) 22340054, Scientific Research(C) 23540348 and Fellows 24340054 from JSPS. This work is also partially supported by the joint research program of the Solar-Terrestrial Environment Laboratory (STEL), Nagoya University, Japan. We want to thank the SciBar and SciBooNe experiments teams for allowing us to use the SciBar as a cosmic ray detector. We are also grateful to the National Institute of Astrophysics, Optics and Electronics (INAOE) for allowing us to continue the experiment at the top of Mt. Sierra Negra. Finally we want to thank Mexican Physics Society and local organizing committee of the 35th ICRC for the funds to attend conference.

\section{References}

[1] J.F. Valdés-Galicia, et.al., Solar neutron events as a tool to study particle acceleration at the Sun, Advances in Space Research 43 (2009) 565-572.

[2] Y. Nagai, et. al., First cosmic-ray measurements by the SciCRT solar neutron experiment in Mexico, Astroparticle Physics 59 (2014) 39-46.

[3] Y.Sasai, et. al., A faster and more reliable data acquisition system for the full performance of the SciCRT, Nuclear Instrument and Methods in Physics 857 (2017) 50-57.

[4] H.Grote, Pattern recognition in high-energy physics, Rep.Prog.Phys. 50 (1987) 473-500.

[5] A.Strandlie, et. al., Track and Vertex Reconstruction: From Classical to Adaptive Methods, Rev. Mod. Phys. 82 (2010) 1419.

[6] R.Mankel, Pattern recognition and event reconstruction in particle physics experiments, Rep.Prog.Phys. 67 (2004) 553-622.

[7] Rafael C. Gonzalez, et.al., Digital Image Processing. Prentice Hall, New Jersey, 2th ed., 2002.

[8] Christian Lippmann, Particle identification, Nuclear Instruments and Methods in Physics Research A 666 (2012) 148-172.

[9] Syed Naeem Ahmed, Physics and Engineering of Radiation Detection. Academic Press, Great Britain, 1th ed., 2007.

[10] Geant4, "Geant4: A toolkit for the simulation of the passage of particles through matter." http://geant 4.cern.ch/, 2017. 